\title{
Pattern of Peripheral Neuropathy Among Patients With Alcohol Dependence Syndrome
}

\author{
John Rebecca ${ }^{1}$, George Peter ${ }^{2}$, Fernandes Kavina ${ }^{3}$ \\ ${ }^{1}$ (Resident, Department Of General Medicine, Father Muller Medical College, India) \\ ${ }^{2}$ (Professor, Department Of General Medicine, Father Muller Medical College, India) \\ ${ }^{3}$ (Senior Resident, Department Of General Medicine, Father Muller Medical College, India)
}

\begin{abstract}
Peripheral neuropathy due to alcohol abuse is common but often difficult to identify in its initial stages. Early identification and initiation of treatment would reduce its progression further limiting the disabilities. The study aims at identifying the pattern of peripheral neuropathy among alcohol dependent patients and grading its severity. The study adopts Toronto Clinical Neuropathy Scoring Scale (TCNS) which utilizes the clinical assessment of symptoms, sensory tests, and lower limb reflexes to find the occurrence of neuropathy and stratify its severity. It was carried out on 30 patients admitted at a tertiary hospital in Southern India with history suggestive of Alcohol Dependence Syndrome. In this study the prevalence of neuropathy was $63.3 \%$. This included $23.3 \%$ with mild neuropathy, $13.3 \%$ with moderate neuropathy and $26.7 \%$ with severe neuropathy. Patients with mild neuropathy were symptomatic but did not have any neurological signs or deficits. In moderate and severe neuropathy, patients showed more clinically detectable signs than symptoms. Total life time dose of alcohol and duration of alcohol consumption did not correlate with severity of neuropathy. TCNS is a simple, cost effective and efficient tool to identify neuropathy and to possibly assess its severity. Sensory complains, sensory deficits and diminished reflexes were observed in alcoholic neuropathy. Studies on larger population groups are recommended to evaluate the pattern of neuropathy in alcohol dependent patients and to compare the same with nerve conduction studies.

Keywords: Alcohol, CAGE questionnaire, Peripheral neuropathy, TCNS, Total life time dose of ethanol.
\end{abstract}

\section{Introduction}

Peripheral neuropathy is the most frequent neurological complication occurring from chronic alcohol abuse. It affects $10-75 \%$ of people with alcoholic abuse. ${ }^{[1]}$ The relative risk of peripheral polyneuropathy in patients with chronic alcohol abuse is $3.9 \% .{ }^{[2]}$ It is characterized by a symmetric polyneuropathy involving the lower extremities with little response to recommended treatment. ${ }^{[3,4]}$ Various factors precipitate alcohol associated neuropathy; these include direct toxicity of alcohol, underlying malnourishment, quantity and duration of alcohol consumption, as well as a familial predisposition. ${ }^{[3]}$ Peripheral neuropathy due to alcoholism is difficult to diagnose clinically in the initial stages. Peripheral nerves have a limited capacity to regenerate, emphasizing the importance of early detection of this disease. ${ }^{[5]}$

This study utilizes Toronto Clinical Neuropathy Scoring Scale (TCNS) to identify neuropathy and grade its severity among patients with alcohol dependence syndrome. ${ }^{[6]}$

\section{Materials and methods}

2.1 Materials: A total of 30 patients admitted at a tertiary hospital in Southern India with history suggestive of Alcohol Dependence Syndrome were included in this study. The descriptive study was performed over a period of 6 months, starting from January 2016.

2.2 Inclusion criteria: Patients who consumed at least $100 \mathrm{ml}$ of ethyl alcohol ( $3 \mathrm{~L}$ of beer or $300 \mathrm{ml}$ of spirits) per day for more than 3 years were recruited for the study. ${ }^{[7]}$

2.3 Exclusion criteria: Those patients with $\mathrm{h} / \mathrm{o}$ diabetes mellitus, occupational exposure to toxins and vibrations, underlying malignancy or receiving chemotherapy, significant trauma to limbs, chronic kidney disease, hypothyroidism or fever, leprosy, shingles or HIV at presentation were excluded.

2.4 Methods: The study utilized the Toronto Clinical Neuropathy Scoring Scale (TCNS) to identify the presence of alcohol related peripheral neuropathy in the patients. The TCNS is a validated tool that has been found to correlate to nerve conduction studies as well as to nerve biopsy findings in patients with diabetic polyneuropathy. ${ }^{[6]}$ The maximum score in TCNS is 19 , derived from clinical assessment of symptoms, sensory tests, and lower limb reflexes. Six scores resulted from recognizing sensory symptoms. They included identifying presence of pain, numbness, tingling and weakness in the foot, presence of similar upper-limb symptom and ataxia. Five points were derived from sensory testing and eight from lower-limb reflexes. Sensory testing was done at great toe for pinprick, temperature, light touch, vibration and position. The reflexes were 
tested on knee and angle. The neuropathy was graded based on the scores from TCNS as follows: 0-5 no neuropathy, 6-8 mild, 9-11 moderate, and 12 or more equated to severe. ${ }^{[8]}$

In addition, patients were screened using the CAGE questionnaire which is a validated tool to identify the presence of alcohol dependence. Developed by Ewing in 1968, the screening test has 4 items, and a score greater than 2 is considered clinically significant. ${ }^{[9,10]}$

The amount of alcohol consumption could correlate with the severity of neuropathy. The total life time dose of ethanol (TLDE) expressed in $\mathrm{kg}$ of ethanol per $\mathrm{kg}$ of body weight, was utilized to identify the same.

Statistical data analysis was carried out using SPSS 8 software. A p-value between 0.01 and 0.05 was set as statistically significant and p-value less than 0.01 was considered to be highly significant.

\section{Results}

The prevalence of neuropathy in the present study was $63.3 \%(\mathrm{n}=19)$. Among the 19 patients with neuropathy, 7 were detected to have mild neuropathy, 4 with moderate neuropathy and 8 with severe neuropathy. The spectrum of alcoholic neuropathy observed in the present study is depicted in Fig 1 .

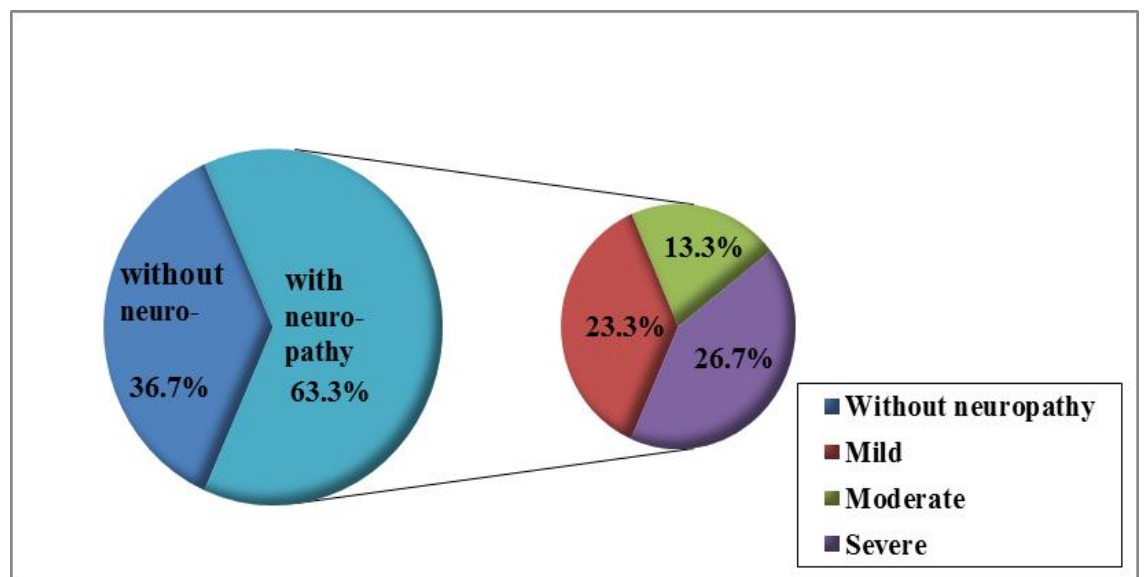

Figure 1: Spectrum of Alcoholic Neuropathy based on TNCS

The details of study population with and without neuropathy are compared in Table 1. The patients with alcohol dependence included in our study were aged between 30-55 years. The average age in the groups without neuropathy and with neuropathy were similar, with the mean ages of $39.9 \pm 7.5$ and $39.5 \pm 17.4$ years respectively. A higher incidence of family history of alcoholism was noted in subjects with neuropathy.

\begin{tabular}{|l|l|l|}
\hline & Without neuropathy & With neuropathy \\
\hline Cases & 11 & 19 \\
\hline Average age (years) & $39.9 \pm 7.5$ & $39.5 \pm 17.4$ \\
\hline Positive family history (\%) & 45.4 & 78.9 \\
\hline
\end{tabular}

Table 1: Demography of patients with and without neuropathy

Table 2 compares average years of alcohol consumption, TLDE and CAGE score among patients with and without neuropathy. The mean alcohol consumption years were $17.0 \pm 6.7$ in subjects without neuropathy. This was similar to those having neuropathy with a mean consumption of $17.4 \pm 6.7$ years. The mean value of TLDE was $19.2 \pm 8.8$ in those without neuropathy and $27.1 \pm 17.1$ in patients of alcoholic neuropathy. The pvalue calculated using Mann-Whitney test did not show statistically significant relation between the TLDE and the occurrence of peripheral neuropathy in this study group. In response to CAGE questionnaire, patients without neuropathy scored $3.18 \pm 0.60$, while those with clinically demonstrable neuropathy scored $3.68 \pm 0.48$. This was statistically significant between patients with and without neuropathy $(\mathrm{p}<0.05)$.

\begin{tabular}{|l|l|l|l|}
\hline & Without neuropathy & With neuropathy & p - value \\
\hline Mean alcoholic intake duration (years) & $17.0 \pm 6.7$ & $17.4 \pm 6.7$ & $>0.05$ \\
\hline $\begin{array}{l}\text { Mean total life time dose of ethanol } \\
\text { (kg ethanol/kg body weight) }\end{array}$ & $19.2 \pm 8.8$ & $27.1 \pm 17.1$ & $>0.05$ \\
\hline $\begin{array}{l}\text { Average CAGE score } \\
\text { (max. score =4) }\end{array}$ & $3.18 \pm 0.60$ & $3.68 \pm 0.48$ & $<0.05$ \\
\hline
\end{tabular}

Table 2: Comparison of alcohol intake duration, dose, CAGE score among patients with and without neuropathy 
The mean value of overall TCNS score of subjects without neuropathy was $2.91 \pm 1.64$. This included scores from clinical symptoms, sensory tests and reflexes. It was correspondingly $9.84 \pm 3.17$ on patients with neuropathy. Further, the clinical symptoms and sensory signs scores as obtained through TCNS were analysed. On a scale of 6 , the mean scores on clinical symptoms in patients with and without neuropathy were $3.05 \pm 1.96$ and $1.09 \pm 0.94$, respectively. The signs score was derived on a scale of 5 . The mean score on sensory signs in patients with and without neuropathy were $2.32 \pm 1.38$ and $1.82 \pm 0.98$.

The variation of clinical symptoms and sensory signs were compared among people with different grades of neuropathy. The mean symptoms score for patients with mild, moderate and severe neuropathy were $3.14 \pm 1.46,0.75 \pm 0.96$ and $4.12 \pm 1.81$, respectively. The corresponding mean values of signs score for patients with mild, moderate and severe neuropathy were $1.86 \pm 0.90,1.0 \pm 0.82$ and $3.37 \pm 1.19$. In some patients, clinical symptoms were more prominent than sensory signs, whereas in others symptoms and signs were comparatively present in equal or lesser grades This was expressed as the percentage of patients having clinical symptoms score greater, equal or less than sensory signs score, as illustrated in Table 3.

\begin{tabular}{|l|l|l|l|}
\hline \multirow{2}{*}{ Grade of neuropathy } & \multicolumn{3}{|c|}{ \% of patients } \\
\cline { 2 - 4 } & $\begin{array}{l}\text { Symptoms score > Signs } \\
\text { score }\end{array}$ & $\begin{array}{l}\text { Symptoms score =Signs } \\
\text { score }\end{array}$ & $\begin{array}{l}\text { Symptoms score < Signs } \\
\text { score }\end{array}$ \\
\hline Mild $(\mathrm{n}=7)$ & $71.4 \%$ & $14.3 \%$ & $14.3 \%$ \\
\hline Moderate $(\mathrm{n}=4)$ & $25 \%$ & 0 & $75 \%$ \\
\hline Severe $(\mathrm{n}=8)$ & $50 \%$ & 0 & $50 \%$ \\
\hline
\end{tabular}

Table 3: Variation of TCNS symptoms and signs scores among different grades of neuropathy

From the above table, we conclude that those patients who had mild neuropathy have more symptoms while they have hardly any sensory signs or deficits. In moderate and severe neuropathy, the patients tend to have more clinically detectable signs than symptoms.

\section{Discussion}

Early identification of alcohol induced peripheral neuropathy caused by chronic alcoholism and timely initiation of its treatment are of utmost importance to prevent chronic disabilities to the patient. Therefore, awareness of physicians has a key role to play in detecting peripheral neuropathy.

Our study identified the prevalence of alcoholic neuropathy to be $63.3 \%$. This was similar to the findings of Ammendola et al. ${ }^{[11]}$ reporting an occurrence of $67.7 \%$. However, in another study, they noticed that the frequency of alcoholic neuropathy was only $30.3 \% .^{[12]}$

Strong family history of alcoholism was observed in patients with alcoholic neuropathy as compared to those without neuropathy. While $78.9 \%$ of patients with neuropathy reported positive family history of alcoholism, it was correspondingly $45.4 \%$ in the group without neuropathy. This is similar to the findings of Ammendola et al. ${ }^{[12]}$ where $36 \%$ of patients without neuropathy had a positive family association of alcoholism and $51 \%$ in patients with neuropathy. Pessione et al. ${ }^{[13]}$ also reported similar observations and suggested that alcohol induced neuropathy may be a marker of hereditary susceptibility to alcoholism.

There are conflicting reports on the role of nutritional deficiency and toxic effect of alcohol in initiating peripheral neuropathy on alcohol dependents. Shattuck proposed that lack of thiamine played an important role in developing neuropathy. ${ }^{[14]}$ Strauss conducted a study on ten patients who continued their daily alcohol consumption along with a well-balanced diet supplemented by yeast and vitamin B concentrates. He concluded that alcoholic neuropathy resulted from dietary deficiency and not from the neurotoxic effects of alcohol. ${ }^{[15]}$ Victor and Adams presented further evidences supporting that alcoholic neuropathy is fundamentally a nutritional disease. ${ }^{[16]}$ On the contrary, Behse and Buchthal reported no relation between nutritional status and neuropathy. ${ }^{[7]}$ Monforte et al. ${ }^{[17]}$ identified that total life time of amount of alcohol and duration of alcohol consumption correlated with neuropathy. ${ }^{[}$Studies by Ammandola et al. ${ }^{[12]}$ and Mellion et al. ${ }^{[18]}$ established significant association between duration of alcohol consumption and cumulative alcohol intake (TLDE) to neuropathy. In the present study, the average duration of alcohol consumption was $17.3 \pm 6.6$ years. The mean total life time dose of alcohol was $24.23 \pm 15.0$. There was no significant difference noted between the groups with and without neuropathy on the basis of total life time dose of ethanol or average alcohol consumption years. This suggests that the toxic dose of alcohol may not be the only factor contributing to alcoholic peripheral neuropathy.

The most frequent symptom, foot pain was seen in $47 \%$ of the study population. Other common symptoms included numbness and tingling sensation were noted in $43 \%$. The most commonly detected sign on neurological examination was loss of light touch sensation and was noted in $73 \%$ of the study population. This was followed by loss of vibration sense, observed in $60 \%$ of subjects. The prevalence of clinical symptoms and sensory signs among different grades of neuropathy were assessed based on their respective scores derived from TCNS. In the present study, $71.4 \%$ of patients detected with mild neuropathy had more presenting symptoms than deficits in the sensory system. In patients with moderate neuropathy, sensory signs were more detectable 
than clinical symptoms and $75 \%$ of them had more sensory signs than symptoms. However, patients with severe neuropathy presented positive symptoms and sensory signs equally. We felt that sensory symptoms are more consistent than signs for diagnosis of alcoholic neuropathy in initial stages.

There was only one female enrolled in this study. She had presented with 5 years of alcohol abuse. She developed moderate neuropathy despite comparatively fewer years of alcohol abuse and low LTDE as compared to males. This also correlates with the findings of Ammendola et al. ${ }^{[11]}$ where females demonstrated lower tolerance to toxic levels of alcohol.

As noted from the study, majority of alcohol dependent patients presented with symptoms of peripheral neuropathy. In patients with severe neuropathy, symptoms, sensory and reflex mechanisms were affected. Along with the duration and quantity of alcohol consumed there may be other modulating factors that lead to neuropathy in alcohol dependent patients. Possible limitations of the study are relatively small sample size and lack of nerve conduction studies. Further studies are recommended to evaluate the effect of nutritional status, genetic predisposition and other factors in perpetuation of peripheral neuropathy in patients with chronic alcohol abuse.

\section{Conclusion}

Peripheral neuropathy is a major complication of alcohol abuse. TCNS is a simple, cost effective and efficient method to ascertain the presence of alcoholic neuropathy and grade its severity. Sensory symptoms, sensory deficits and diminished reflexes are seen in patients with alcoholic neuropathy. Studies on larger population groups are recommended for better understanding of the pattern of peripheral neuropathy.

\section{Compliance with Ethical Standards}

Ethical approval: This article does not contain any data obtained from any other source/ or authors.

Source of Support: No funding obtained for this study.

Conflict of Interest: No conflicts of interest.

\section{References}

[1]. Daroff RB, Jankovic J, Mazziotta JC, Pomeroy SL. Bradley's neurology in clinical practice.; $7^{\text {th }}$ ed. United States of America: Elsevier Health Sciences; 2015

[2]. Hanewinckel R, Oijen M, Ikram A, Doom PA. The epidemiology and risk factors of chronic polyneuropathy. Eur J Epidemiol 2016; 31:5-20.

[3]. Chopra K, Tiwari J. Alcoholic neuropathy: possible mechanisms and future treatment possibilities. Br J Clin Pharmacol 2011; 73(3):348-62.

[4]. Koike H, Sobue G. Alcoholic neuropathy. Curr Opin Neurol 2006; 19(5):481-6.

[5]. Ropper A, Samuels M, Klein J. Adams and Victor's priciples of Neurology; $10^{\text {th }}$ ed. United States of America: McGraw Hill professional; 2014

[6]. Bril V, Perkins BA. Validation of the Toronto clinical scoring system for diabetic polyneuropathy. Dia Care 2002; 25(13):2048-52.

[7]. Behse F, Buchthal F. Alcoholic neuropathy: clinical, electrophysiological and biopsy findings. Ann Neurol 1977; 2: 95-110.

[8]. Bril V, Tomioka S, Perkins BA. Reliability and validity of the modified Toronto clinical neuropathy score in diabetic sensorimotor polyneuropathy. Diabet Med 2009; 26:240-6.

[9]. Ewing JA. Detecting alcoholism: The CAGE questionnaire. JAMA 1968 252:1905-7.

[10]. Dhalla S, Kopec JA. The CAGE questionnaire for alcohol misuse: a review of reliability and validity studies. Clin Invest Med 2007; 30(1):33-41.

[11]. Ammendola A, Gemini D, Iannacconne S, Argenzio F, Ciccone G, Ammendola E et al. Gender and peripheral neuropathy in chronic alcoholism: a clinical-electroneurographic study. Alcohol and alcoholism 2000; 35(4):368-71.

[12]. Ammendola A, Tata R, Aurilio C, Ciccone G, Gemini D, Ammendola E et al. Peripheral neuropathy in chronic alcoholism: a retrospective crosssectional study in 76 subjects 2001; 36(3):271-75.

[13]. Pessione F, Gerchstein JK, Rueff B. Parental history of alcoholism: a risk factor for alcohol-related peripheral neuropathies. Alcohol Alcohol 1995; 30:749-54.

[14]. Shattuck GC. The relation of beri-beri to polyneuritis from other causes. Am J Trop Med 1928; 8:539-43

[15]. Strauss MB. The etiology of alcoholic polyneuritis. Am J Med Sci 1935; 189:378-82.

[16]. Victor M, Adams RD. on the etiology of alcoholic neurologic diseases with special reference to the role of nutrition. Am J Clin Nutr 1961; 9:379-97.

[17]. Monforte R, Estruch R, Valls-Sole J, Nicholas J, Villalta J, Urnamo-Marquez A. Autonomic and peripheral neuropathies in patients with chronic alcoholism: a dose-related toxic effect of alcohol. Arch Neurol 1995; 52:45-51.

[18]. Mellion M, Gilchrist JM, DelaMonte S. Alcohol-related peripheral neuropathy: nutritional, toxic, or both?. Muscle Nerve 2011; 43(3):309-16. 